\title{
NONOSCILLATION THEOREMS FOR A NONLINEAR DIFFERENTIAL EQUATION ${ }^{1}$
}

\author{
H. E. GOLLWITZER
}

\begin{abstract}
This paper is concerned with the problem of specifying growth conditions on the positive function $q(t)$ which imply that all solutions of the nonlinear second order ordinary differential equation $y^{\prime \prime}+q(t)|y|^{\alpha} \operatorname{sgn} y=0, \alpha>0$, are nonoscillatory on a half line. Several different results are given, and the usual explicit monotonicity condition on $q$ has been avoided to a certain degree.
\end{abstract}

In the real, nonlinear differential equation

$$
y^{\prime \prime}+q(t)|y|^{\alpha} \operatorname{sgn} y=0, \quad\left({ }^{\prime}=d / d t\right),
$$

let $q(t)$ be positive, continuous and locally of bounded variation on a half line $(a, \infty)$, and suppose that $0<\alpha \neq 1$. Our purpose here is to give several sets of conditions which imply that all solutions of (1) are nonoscillatory. These conditions are of interest since the usual explicit monotonicity condition on $q$ can be avoided in some sense.

We begin with some preliminary facts and definitions. A realvalued function $f(t)$ is said to be locally of bounded variation on $[a, \infty)$ if $f(t)$ is of bounded variation on each compact subinterval. A real-valued function $f(t)$ is said to be of class $\operatorname{CBV}_{\text {loc }}[a, \infty)$, $f \in \mathrm{CBV}_{\text {loo }}[a, \infty)$, if $f$ is continuous and locally of bounded variation on the half line $[a, \infty)$. If $f \in \mathrm{CBV}_{\text {loc }}[a, \infty), \tau \in[a, \infty)$, then $f$ has the representation $f(t)=f(\tau)+f_{+}(t, \tau)-f_{-}(t, \tau), t \in[a, \infty)$, where where $f_{+}, f_{-}$, for fixed $\tau$, are continuous, nonincreasing in $t$ for $a \leqq t \leqq \tau$, and nondecreasing in $t$ for $t \geqq \tau$. This representation is called the Jordan decomposition of $f$ (see [7, Chapter 2]). Throughout the paper we will assume that the function $q$ defined in (1) is positive and of class $\mathrm{CBV}_{\text {loc }}[a, \infty)$. A solution of (1) with real initial values is real-valued and exists on $[a, \infty),[2],[5],[9]$. Furthermore, if $y(t)$ is a solution of (1) then $-y(t)$ is also a solution. A nontrivial solution of (1) is called oscillatory if it has arbitrarily large zeros. A nontrivial solution of (1) which is eventually of one sign is called nonoscillatory, and (1) is called nonoscillatory if every nontrivial solution

Received by the editors September 11, 1969.

AMS 1968 subject classifications. Primary 3442.

Key words and phrases. Nonoscillation, nonlinear, second order, bounded variation, Riemann-Stieltjes integral. 9575.

1 This work was supported in part by National Science Foundation grant GP- 
is nonoscillatory. Further results concerning (1) are given in [1], [6], [10], [12].

For purposes of comparison and convenience we will first state the major results of the paper. In all of the results given here we assume that $q$ has the Jordan representation $q(t)=q(a)+q_{+}(t, a)$ $-q_{-}(t, a), t \geqq a$. All integrals displayed in these results are improper Riemann-Stieltjes integrals.

Theorem 1. In (1) let $\alpha>1, q(t) \in \mathrm{CBV}_{\text {loc }}[a, \infty), q(t)>0$ for $t \geqq a$. Assume that

$$
\int_{a}^{\infty} s^{\alpha-1} q(s) d s<\infty, \quad \int_{a}^{\infty} q(s)^{-1} d q_{+}(s, a)<\infty .
$$

If

$$
\lim _{t \rightarrow \infty} t \int_{t}^{\infty} s^{\alpha-1} q(s) d s=0,
$$

then (1) is nonoscillatory.

TheOREM 2. In (1) let $\alpha>1, q(t) \in \mathrm{CBV}_{\text {loc }}[a, \infty), q(t)>0$ for $t \geqq a$. Assume that

$$
\int_{a}^{\infty} q(s)^{2 /(\alpha+1)} d s<\infty, \quad \int_{a}^{\infty} q(s)^{-1} d q_{+}(s, a)<\infty .
$$

If

$$
\lim _{t \rightarrow \infty} t \int_{t}^{\infty} q(s)^{2 /(\alpha+1)} d s=0,
$$

then (1) is nonoscillatory.

Theorem 3. In (1) let $\alpha>1, q(t) \in \operatorname{CBV}_{\text {loo }}[a, \infty), q(t)>0$ for $t \geqq a$. If

$$
\int_{a}^{\infty} q(s)^{1 /(\alpha+1)} d s<\infty, \quad \int_{a}^{\infty} q(s)^{-1} d q_{+}(s, a)<\infty,
$$

then (1) is nonoscillatory.

Theorem 4. In (1) let $0<\alpha<1, q(t) \in \operatorname{CBV}_{\text {loc }}[a, \infty), q(t)>0$ for $t \geqq a$. Assume that

$$
\int_{a}^{\infty} s^{\alpha} q(s) d s<\infty, \quad \int_{a}^{\infty} q(s)^{-1} d q_{+}(s, a)<\infty .
$$


If

$$
\lim _{t \rightarrow \infty} q(t)^{(\alpha-1) / 2} \int_{t}^{\infty} s^{\alpha} q(s) d s=0,
$$

then (1) is nonoscillatory.

THEOREM 5. In (1) let $0<\alpha<1, q(t) \in \mathrm{CBV}_{\mathrm{loc}}[a, \infty), q(t)>0$ for $t \geqq a$. Assume that

$$
\int_{a}^{\infty} q(s)^{1 /(\alpha+1)} d s<\infty, \quad \int_{a}^{\infty} q(s)^{-1} d q_{+}(s, a)<\infty .
$$

If

$$
\lim _{t \rightarrow \infty} q(t)^{(\alpha-1) / 2(\alpha+1)} \int_{t}^{\infty} q(s)^{1 /(\alpha+1)} d s=0,
$$

then (1) is nonoscillatory.

TheOREM 6. In (1) let $0<\alpha<1, q(t) \in \mathrm{CBV}_{\text {loc }}[a, \infty), q(t)>0$ for $t \geqq a$. If

$$
\int_{a}^{\infty} s q(s) d s<\infty, \quad \int_{a}^{\infty} q(s)^{-1} d q_{+}(s, a)<\infty,
$$

then (1) is nonoscillatory.

Before proceeding any further, we must explain the significance of the integral condition

$$
\int_{a}^{\infty} q(s)^{-1} d q_{+}(s, a)<\infty .
$$

As a consequence of the additivity properties of the total variation of $q$, it is clear that if (12) holds, then $\int_{\tau}^{\infty} q(s)^{-1} d q_{+}(s, \tau)<\infty$ for any fixed $\tau \geqq a$. If $q$ is a monotone decreasing function then (12) holds. Furthermore, it was shown in [4] that if (12) holds, then $q$ is of bounded variation on $[a, \infty)$. The following lemma gives some indication of the type of function $q$ which satisfies (12). A proof of this lemma was given in [4].

Lemma 1. Let $b(t), c(t)$ be positive, continuous and of bounded variation on $[a, \infty)$. If $b(t) \geqq \delta>0$, and $c(t)$ is nonincreasing, then (12) holds.

We note that Theorem 1 gives an independent proof of the wellknown result of Atkinson [1]. For if $q$ is continuously differentiable, 
decreasing and $\int^{\infty} s^{\alpha} q(s) d s<\infty$, then (2), (3) hold. Theorems 1, 2, and 3 are approximately of the same degree of generality. For if $q(t)=t^{-\sigma}$, then a simple calculation shows that we must have $\sigma>\alpha+1$. Consequently these results are not as sharp as those given by Kiguradze [10], Nehari [12] and Coffman and and Wong [2] in the case when $q$ is a monotone function. Theorem 6 generalizes a recent result of Heidel [6] in that we have removed an explicit monotonicity condition on $q$. The results given in Theorems 4,5 and 6 are also approximately of the same degree of generality. For if $q(t)=t^{-\sigma}$, then a simple calculation shows that we must have $\sigma>2$.

The proofs of these theorems are preceded by an important lemma.

Lemma 2. In the differential equation

$$
y^{\prime \prime}+q(t)|y|^{\alpha} \operatorname{sgn} y=0,
$$

let $0<\alpha$ and assume that $q(t) \in \mathrm{CBV}_{\mathrm{loc}}[a, \infty), q(t)>0$ for $t \geqq a$. Let $\tau \in[a, \infty)$ be fixed but arbitrary and let $q(t)=q(\tau)+q_{+}(t, \tau)-q_{-}(t, \tau)$ be the Jordan decomposition of $q$ for $t \in[a, \infty)$. Then for $t \in[a, \infty)$,

$$
\begin{aligned}
& E(\tau) \exp \left\{-\int_{\tau}^{t} q(s)^{-1} d q_{-}(s, \tau)\right\} \\
& \leqq E(t) \leqq E(\tau) \exp \left\{\int_{\tau}^{t} q(s)^{-1} d q_{+}(s, \tau)\right\}, \\
& B(\tau) \exp \left\{-\int_{\tau}^{t} q(s)^{-1} d q_{+}(s, \tau)\right\} \\
& \leqq B(t) \leqq B(\tau) \exp \left\{\int_{\tau}^{t} q(s)^{-1} d q_{-}(s, \tau)\right\},
\end{aligned}
$$

where

$$
E(t) \equiv y^{\prime}(t)^{2} / 2+(\alpha+1)^{-1} q(t)|y(t)|^{\alpha+1}, \quad B(t)=q(t)^{-1} E(t) .
$$

This lemma is similar to a special case of a result which was announced by Izjumova and Kiguradze [9]. A different proof of this lemma was given in [4], where we used integral inequalities for Riemann-Stieltjes integrals. As was noted in [4], the estimates given in Lemma 2 are not valid if the continuity condition on $q$ is relaxed. This follows from the fact that $q(t)$ and $q_{+}(t, a), q_{-}(t, a)$ are, in general, simultaneously continuous or discontinuous, and hence the integrals in (13), (14) do not exist. Techniques for partially circumventing this problem were outlined in [4]. However, since nothing new seems to accrue in this case, we have omitted it here. 
Proofs of Theorems 1 and 2. If $\alpha>1$ and $y(t)$ is any nontrivial solution of (1), then $y(t)$ is also a solution of the linear equation

$$
u^{\prime \prime}+u q(t)|y(t)|^{\alpha-1}=0 .
$$

As a consequence of (2), we see from (13) that $y^{\prime}(t)$ is bounded on $[a, \infty)$. Hence $|y(t)| \leqq C t$ for large $t$ and some constant $C$. We have taken $\tau=a$ in (13). Since (3) holds, and hence

$$
\limsup _{t \rightarrow \infty} t \int_{t}^{\infty} q(s)|y(s)|^{\alpha-1} d s \leqq \limsup _{t \rightarrow \infty} t \int_{t}^{\infty}(C s)^{\alpha-1} q(s) d s=0,
$$

a well-known theorem of Hille [8, p. 246] applies and therefore (15), and consequently (1), is nonoscillatory. The proof of Theorem 1 is complete.

The proof of Theorem 2 proceeds in exactly the same manner. If (4) holds, then from (13) it follows that $|y(t)|^{\alpha-1} \leqq C q(t)^{(1-\alpha) /(1+\alpha)}$ for some constant $C$. The arguments used in the proof of Theorem 1 apply here also and hence (1) is nonoscillatory. This completes the proof of Theorem 2.

Proof of Theorem 3. The proof of Theorem 3 follows the original argument given by Atkinson [1]. Assume, for the sake of contradiction, that $y(t)$ is an oscillatory solution of (1). Since solutions are unique, there is a sequence of zeros $\left\{t_{n}\right\}$ of $y(t)$ such that $y^{\prime}\left(t_{n}\right)>0$, $t_{n} \rightarrow \infty$. Let $t=s_{n}>t_{n}$ be the first value of $t$ for which the derivative vanishes. In (13), with $\tau=t_{n}$, we have the estimate

$$
2 q(t)|y(t)|^{\alpha+1} \leqq y^{\prime}\left(t_{n}\right)^{2} \exp \left\{\int_{t_{n}}^{t} q(s)^{-1} d q_{+}\left(s, t_{n}\right)\right\} .
$$

We use this estimate together with the arguments of Atkinson to complete the proof of Theorem 3.

An examination of the proof of Theorem 3 shows that the result also holds when $\alpha=1$. In this case we have a generalization of a theorem of Leighton [11, p. 43].

Proof of THEOREM 4. The proof of this theorem is similar to the one given by Atkinson [1], but the techniques are different. Assume, for the sake of contradiction, that $y(t)$ is a nontrivial oscillatory solution of (1) with $0<\alpha<1$. There must be a sequence of zeros $\left\{t_{n}\right\}$ of $y(t)$ with $y^{\prime}\left(t_{n}\right)>0, t_{n} \rightarrow \infty$. For if $y^{\prime}\left(t_{n}\right)=y\left(t_{n}\right)=0$, then from (13), with $\tau=t_{n}$, it follows that $y(t) \equiv 0$ and this is a contradiction. If $t_{n} \nrightarrow \infty$, there must be a subsequence of $\left\{t_{n}\right\}$ converges to a finite value $c$. By Rolle's theorem there must also be a sequence $\left\{\tau_{k}\right\}$ such that $\tau_{k} \rightarrow c, y^{\prime}\left(\tau_{k}\right)=0$. Since all solutions of (1) exist on $[a, \infty)$, we see, by 
continuity, that $y(c)=y^{\prime}(c)=0$. But this contradicts the fact that $y(t) \not \equiv 0$.

Let $t=s_{n}>t_{n}$ be the first value of $t$ for which $y^{\prime}(t)$ vanishes. From (1), $y^{\prime \prime}(t) \leqq 0$ on $\left[t_{n}, s_{n}\right]$. Two integrations of this inequality over $\left[t_{n}, t\right]$ show that $y(t) \leqq y^{\prime}\left(t_{n}\right) t$ on $\left[t_{n}, s_{n}\right]$. If we use this estimate for $y(t)$ and integrate (1), then

$$
y^{\prime}\left(t_{n}\right)=\int_{t_{n}}^{s_{n}} q(s)|y(s)|^{\alpha} d s \leqq y^{\prime}\left(t_{n}\right)^{\alpha} \int_{t_{n}}^{\infty} s^{\alpha} q(s) d s .
$$

However, from (14) and the second integral condition in (7), it follows that

$$
y^{\prime}\left(t_{n}\right)^{2} \geqq C^{2} q\left(t_{n}\right),
$$

where $C$ is a positive constant which does not depend on $t_{n}$. We have taken $\tau=a$ in (14) to obtain this estimate. Combining (17) and (18) we obtain

$$
C \leqq q\left(t_{n}\right)^{(\alpha-1) / 2} \int_{t_{n}}^{\infty} s q(s) d s .
$$

Since $t_{n} \rightarrow \infty$ and (8) holds, this inequality eventually leads to a contradiction and hence (1) is nonoscillatory. The proof of Theorem 4 is complete.

The proof of Theorem 5 is similar and will not be given here.

Proof of TheOREM 6 . This theorem is a slight generalization of a result of Heidel [6]. Assume, for the sake of contradiction, that $y(t)$ is an oscillatory solution of (1) with zeros at $t=t_{n}, t_{n} \rightarrow \infty$. Let $t=s_{n}$ be the unique point in $\left[t_{n}, t_{n+1}\right]$ where $|y(t)|$ attains its maximum. Since (11) holds, we can use (14) (here we take $\tau=a$ ) to assert that $\lim _{\inf _{n \rightarrow \infty}}\left|y\left(s_{n}\right)\right|>0$. However, Heidel [6] has shown that if $\int s q(s) d s<\infty$ and $y(t)$ is an oscillatory solution of (1), then $\lim _{t \rightarrow \infty} y(t)$ $=0$. Hence we have a contradiction and (1) is nonoscillatory. The theorem is complete.

\section{REFERENCES}

1. F. V. Atkinson, On second-order non-linear oscillations, Pacific J. Math. 5 (1955), 643-647. MR 17, 264.

2. C. V. Coffman and D. F. Ullrich, On the continuation of solutions of a certain non-linear differential equation, Monatsh. Math. 71 (1967), 385-392. MR 37 \#3078.

3. C. V. Coffman and J. S. W. Wong, On a second order nonlinear oscillation problem, Carnegie-Mellon University Report 69-2.

4. H. E. Gollwitzer, Nonlinear second order differential equations and Stieltjes integrals, University of Tennessee Report, April, 1969. 
5. S. P. Hastings, Boundary value problems in one differential equation with a discontinuity, J. Differential Equations 1 (1965), 346-369. MR 31 \#4954.

6. J. W. Heidel, $A$ nonoscillation theorem for a nonlinear second order differential equation, Proc. Amer. Math. Soc. 22 (1969), 485-488.

7. T. H. Hildebrandt, Introduction to the theory of integration, Pure and Appl. Math., vol. 13, Academic Press, New York, 1963. MR 27 \#4900.

8. E. Hille, Non-oscillation theorems, Trans. Amer. Math. Soc. 64 (1948), 234-252. MR 10, 376.

9. D. V. Izjumova and I. T. Kiguradze, Some remarks on the solutions of the equation $u^{\prime \prime}+a(t) f(u)=0$, Differencial'nye Uravnenija 4 (1968), 589-605. (Russian) MR 37 \#3128.

10. I. T. Kiguradze, $O n$ the conditions for oscillation of solutions of the equation $u^{\prime \prime}+a(t)|u|{ }^{n} \operatorname{sgn} u=0$, Časopis Pěst. Mat. 87 (1962), 492-495. (Russian) MR 31 \#6026.

11. W. Leighton, On self-adjoint differential equations of second order, J. London Math. Soc. 27 (1952), 37-47. MR 13, 745.

12. Z. Nehari, A nonlinear oscillation problem, J. Differential Equations 5 (1969), 452-460. MR 38 \#3514.

The University of Tennessee, Knoxville, Tennessee 37916 